\title{
Prognostic significance of LKB1 promoter methylation in cutaneous malignant melanoma
}

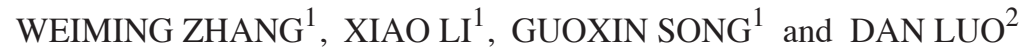 \\ Departments of ${ }^{1}$ Pathology and ${ }^{2}$ Dermatology, \\ The First Affiliated Hospital of Nanjing Medical University, Nanjing, Jiangsu 210029, P.R. China
}

Received October 28, 2015; Accepted April 13, 2017

DOI: $10.3892 / 01.2017 .6431$

\begin{abstract}
Liver kinase B1 (LKB1) loss is a common occurrence in various types of human cancer, and promoter methylation has been hypothesized to be a major mechanism of LKB1 inactivation. The association between LKB1 gene promoter methylation status and tumor progression in cutaneous malignant melanoma (CMM) remains unknown. In the present study, the methylation status of the LKB1 promoter region was examined in 57 human cutaneous malignant melanomas and 50 benign skin lesion controls by methylation-specific polymerase chain reaction. Consequently, 12 (12/57) melanoma tissues exhibited LKB1 promoter methylation, while only 2 (2/50) benign lesions presented with LKB1 hypermethylation. The frequency of LKB1 promoter methylation in melanoma was significantly increased compared with the benign controls $(\mathrm{P}<0.05)$. Additional statistical analysis demonstrated that hypermethylation of the LKB1 gene was correlated with Breslow's thickness, presence of ulceration and American Joint Committee on Cancer stage $(\mathrm{P}<0.05)$. Additionally, Kaplan-Meier analysis revealed that LKB1 hypermethylation was significantly associated with poorer survival $(\mathrm{P}<0.01)$. Multivariate COX regression analysis indicated that LKB1 promoter methylation was an independent prognostic factor for overall survival in patients with melanoma.
\end{abstract}

\section{Introduction}

Cutaneous malignant melanoma (CMM) is a type of skin cancer that originated from uncontrolled proliferation of melanocytes. CMM accounts for $80 \%$ of mortalities arising from skin cancer (1). Although the incidence of melanoma is low, the number of new melanoma cases has increased steadily in the recent years (2). The prognosis of melanoma remains poor

Correspondence to: Professor Dan Luo, Department of Dermatology, The First Affiliated Hospital of Nanjing Medical University, 300 Guangzhou Road, Nanjing, Jiangsu 210029, P.R. China

E-mail: luodanjsph@126.com

Key words: melanoma, prognosis, liver kinase B1, methylation due to its high potential for invasion and metastasis, and its resistance to conventional radiotherapy and chemotherapy (3). Therefore, identifying novel molecular markers for the assessment of prognoses of cutaneous malignant melanoma, or as novel targeted therapeutic agents, will be beneficial for patients with melanoma.

The liver kinase B1 gene (LKB1), also known as STK11, was originally identified as the causative gene of Peutz-Jeghers syndrome, an autosomal dominant inherited disorder characterized by mucosal gastrointestinal polyps and an increased risk of malignant tumors (4). As a tumor suppressor gene, LKB1 suppresses tumor cell growth, induces cell death and inhibits tumor cell metastasis (5). Several studies have demonstrated that the loss of LKB1 promotes metastasis in a number of tumor cell types, including melanoma cells (6-8). At present, LKB1 inactivation mechanisms remain controversial. LKB1 mutations were detected in certain types of sporadic cancers, including lung adenocarcinoma and cervical carcinoma (9). However, only $10 \%$ of patients with cutaneous melanoma have reported to exhibit mutations in the LKB1 gene, which means that genetic mutation is not the major mechanism for LKB1 gene inactivation, and there may be other mechanisms that mediate the lack of LKB1 expression (10-11). Epigenetic alteration of tumor suppressor genes by aberrant methylation in promoter $\mathrm{CpG}$ islands has been acknowledged as an important mechanism for inactivation of gene expression (12-16). Previously, several studies have identified that hypermethylation of the LKB1 promoter region may be detected in certain cancer cell lines and primary tumor samples. Correspondingly, LKB1 transcripts were not detected, and treatment with the demethylating agent 5-aza-2'-deoxycytidine was able to restore LKB1 gene expression in these cells (17). Whether epigenetic alteration of the LKB1 gene leads to an inactivation of LKB1 and a lack of LKB1 expression in melanoma remains unclear.

The aim of the present study was to investigate the status of LKB1 promoter methylation in human cutaneous melanoma and to evaluate the associations between aberrant LKB1 promoter methylation with clinicopathological features and prognosis in patients with melanoma.

\section{Materials and methods}

Tissue specimen preparations. A total of 57 cases of melanoma and 50 cases of benign lesion controls (including dysplastic 
nevi and common nevi, the ratio of males:females was $32 / 25$, mean age of all patients was $59.3 \pm 0.4$.) were selected from archived formalin-fixed paraffin embedded (FFPE) tissue blocks from The First Affiliated Hospital of Nanjing Medical University (Nanjing, China) between January 2003 and December 2010. Prior to sectioning, all archived tissue slides were reviewed by two experienced pathologists using a multi-head light microscope (Nikon $80 i$; Nikon Corporation; Tokyo, Japan). The tumors were classified according to the American Joint Committee on Cancer (AJCC) staging system (18-19). Melanoma Breslow's thicknesses (20) were divided into 2 categories $(<$ or $=2.00$, and $>2.00 \mathrm{~mm}$ ). Detailed clinicopathological parameters, summarized in Table I, were obtained from the medical records or pathology examination reports of patients. The follow-up time was from the date of diagnosis to the date of mortality or the end of follow-up (5 years). Identifiable patient data (name and other private data) were anonymized during the study process. The ethics committee of The First Affiliated Hospital of Nanjing Medical University approved the study design, including the use of all tissue blocks from patients.

Immunohistochemistry (IHC) detection. The level of LKB1 expression was detected by IHC in 57 FFPE melanoma and 50 benign control tissues. Standard immunohistochemical protocol was used. Paraffin (thickness, $4 \mu \mathrm{m}$ ) sections from each specimen were heated at $70^{\circ} \mathrm{C}$ for $60 \mathrm{~min}$ and then deparaffinized with xylene. The sections were hydrated with a gradient of ethanol and immersed in $10 \mathrm{mM} \mathrm{pH} 6.0$ boiling citrate buffer in an autoclave for $15 \mathrm{~min}$ for antigen retrieval. Subsequent to blocking with goat serum for $10 \mathrm{~min}$ at room temperature, the slides were incubated with a monoclonal rabbit anti-LKB1 antibody (cat. no. ab15095; dilution, 1:250; Abcam, Cambridge, UK) overnight at $4^{\circ} \mathrm{C}$ in a humidified chamber. The slides were rinsed, and then incubated with ready-to-use horseradish peroxidase-conjugated universal secondary antibody (cat. no. K5007; Dako; Agilent Technologies, Inc., Santa Clara, CA USA) for $15 \mathrm{~min}$ at room temperature. The chromogenic reaction was developed by incubating the slides with 3,3'-diaminobenzidine and counterstained with hematoxylin for $3 \mathrm{~min}$. The slides were also incubated with non-immune serum (cat. no. ab7481; Abcam) overnight at $4^{\circ} \mathrm{C}$ as negative controls. LKB1 staining was distributed in the nucleus and cytoplasm, and scored by two pathologists based on the percent of positively stained cells and staining intensity. The percentage of positive staining was scored as: $0,0 \% ; 1,0-20 \%$; 2, 20-50\%; or 3, $>50 \%$, and the intensity as: 0 , no staining; 1 , weak staining and visible at high magnification (x400; Nikon $50 i$, Nikon Corporation); 2 , moderate staining and visible at low magnification (x100) and 3, dark staining, visible at low magnification. The total immunostaining score was calculated by multiplying the staining intensity score (range, 0-9) by the score for the percentage of positive staining, as described previously (21). The specimens can be classified into two groups based on LKB1 expression: Low expression (score, 0-3) and high expression (score, 4-9).

Extraction of nucleic acids and quantitative reverse transcription polymerase chain reaction ( $R T$ - $q P C R)$. QIAamp DNA FFPE Tissue kit (cat. no. 56404) was used to isolate
Table I. Liver kinase B1 promoter methylation in melanoma and benign skin lesions.

\begin{tabular}{lccc}
\hline & \multicolumn{2}{c}{ Methylation } & \\
\cline { 2 - 3 } Group & Positive & Negative & P-value \\
\hline Melanoma tissue & 12 & 45 & $<0.05$ \\
Benign skin lesion & 2 & 48 & \\
\hline
\end{tabular}

genomic DNA from FFPE tissues. The hematoxylin and eosin stained slides were first reviewed by pathologists, and the areas of interest were circled to guide macro-dissection of the tumor tissue. In total, $\leq 8$ deparaffinized, unstained sections (thickness, 5-10 $\mu \mathrm{m}$ ) were scraped in a polypropylene micro-centrifuge tube, and subsequently processed according to the manufacturer's instructions (22). The quality and integrity of the DNA were measured by electrophoresis on $0.8 \%$ agarose gels. The DNA samples were quantified spectrophotometrically and stored at $-20^{\circ} \mathrm{C}$ for subsequent testing.

Total RNA from melanoma tissues were extracted using the QIAamp RNeasy FFPE kit (cat no. 73504; Qiagen, Inc., Valencia, CA, USA), The isolation was performed according to the manufacturer's protocol. RNA purity was assessed using the A260/A280 ratio with a SmartSpec Plus spectrophotometer (Bio-Rad Laboratories, Inc., Hercules, CA, USA). Total RNA $(1 \mu \mathrm{g})$ was reverse transcribed into cDNA using the QIAamp QuantiTect Reverse Transcription kit (cat no. 205313; Qiagen, Inc.). RT-qPCR reaction was performed with a QuantiNova SYBR Green RT-PCR kit (cat no. 208152; Qiagen, Inc.) using the Agilent 3000 Real-Time PCR system (Agilent Technologies, Inc.). Human LKB1 and GAPDH primers were as follows: LKB1 forward, 5'-AGGGATGCTTGAGTACG AACC-3' and reverse, 5'-GTCCTCCAAGTACGGCACC-3'; GAPDH forward, 5'-CAAGATCATCAGCAATGCCT-3' and reverse, 5'-TCATGAGTCCTTCCACGATAC-3'. GAPDH was used as an internal control. The relative mRNA expression of LKB1 was normalized to GAPDH and calculated by using the comparative cycle threshold (Ct) method (23). All experiments were replicated three times.

Methylation-specific PCR. Methylation-specific PCR (MSP) was used to detect promoter methylation. The QIAamp EpiTect Fast DNA Bisulfite kit (cat. no. 59824; Qiagen, Inc.) was used to convert DNA samples ( $\geq 2 \mu \mathrm{g}$ ). The sequences of the unmethylated template primers were: forward, 5'-TTTGTGTTTTGATGTTTTAGGTTTTTGT-3' and reverse primer, 5'-AACTCCACACTCTTCCAAAAACAAAACA-3'. The sequences of the methylated template primers were: Forward, 5'-TTTCGACGTTCGTAGGTTTTCGC-3' and reverse, 5'-GCACTCTTCCGAAAACGAAACG-3'. The PCR amplification conditions were as follows: An initial denaturation step at $95^{\circ} \mathrm{C}$ for $5 \mathrm{~min}$, followed by 35 cycles at $94^{\circ} \mathrm{C}$ for $50 \mathrm{sec}$, $59^{\circ} \mathrm{C}$ for $50 \mathrm{sec}, 72^{\circ} \mathrm{C}$ for $50 \mathrm{sec}$ and a final extension at $72^{\circ} \mathrm{C}$ for $10 \mathrm{~min}$.

Statistical analysis. Statistical analyses were performed using SPSS version 19.0 (IBM Corp., Armonk, NY, USA) software. 

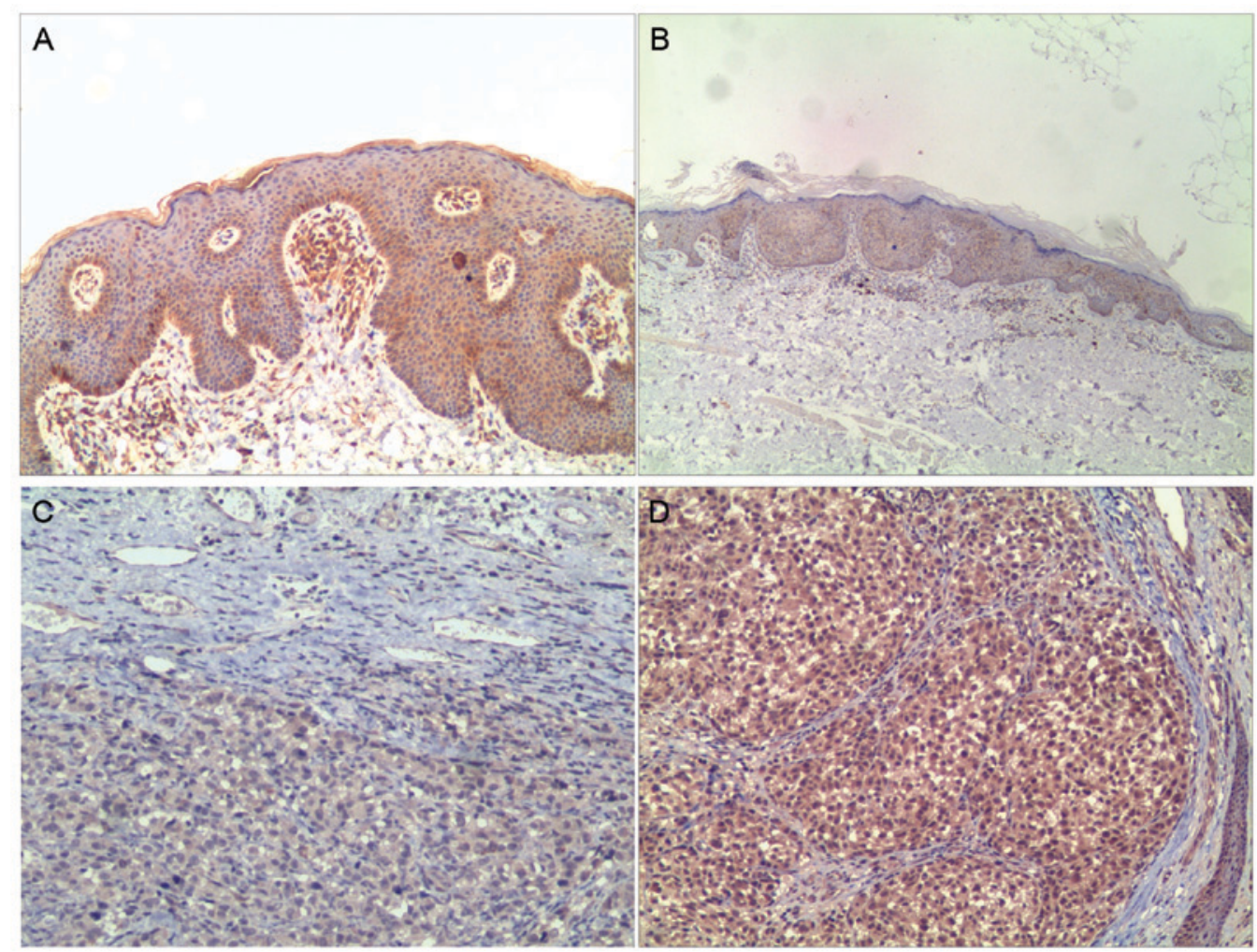

Figure 1. Representative images of LKB1 expression in melanoma tissues and benign skin lesion tissues as detected by immunohistochemical staining. (A) Dark LKB1 staining identified in benign skin lesion tissue. Magnification, x100. (B) Weak LKB1 staining identified in benign skin lesion tissue. Magnification, x100. (C) Moderate LKB1 staining identified in LKB1-methylated melanoma tissue. Magnification, x200. (D) Dark LKB1 staining identified in LKB1 unmethylated melanoma tissue. Magnification, x200. LKB1, liver kinase B1.

Comparisons of LKB1 methylation status with clinicopathological characteristics were made using contingency table $\chi^{2}$ test. The Spearman rank correlation was used to analyze the LKB1 expression at mRNA and protein levels with LKB1 methylation status. Survival curves were estimated using the Kaplan-Meier method, and the log-rank test was used to compare the difference. Multivariate analysis was performed using the Cox proportional hazard models with a confidence interval of 95\% to examine whether LKB1 promoter methylation was an independent prognostic factor for 5-year overall survival rates (OS). The Kruskal-Wallis test was used to compare LKB1 expression between melanoma and benign lesions, methylated melanoma and the unmethylated. All P-values were two-sided, and $\mathrm{P}<0.05$ was considered to indicate a statistically significant difference.

\section{Results}

LKB1 promoter methylation status in human cutaneous melanoma tissues. LKB1 promoter methylation was performed successfully in all cases. It was identified that $12 / 57$ cases exhibited methylation amplification in melanoma tissues, while 2/50 benign controls displayed LKB1 promoter methylation. The frequency of LKB1 promoter methylation was significantly increased in melanoma tissues compared with benign skin controls $\left(\chi^{2}=6.747, \mathrm{P}<0.05\right.$; Table I $)$.

LKB1 promoter methylation is correlated with LKB1 mRNA and protein expression. RT-qPCR was performed successfully in $50(50 / 57)$ melanoma tissues. LKB1 mRNA expression level (LKB1/GAPDH) in the methylated melanoma tissues (range, 0.112-2.524; mean, 0.675) was reduced compared with the unmethylated melanoma tissues (range, 0.522-3.311; mean, 1.541; $\mathrm{P}<0.05)$. In melanoma tissues, LKB1 mRNA expression was correlated with LKB1 promoter methylation status $(\mathrm{r}=0.354 ; \mathrm{P}<0.05$; data not shown).

LKB1 protein expression was localized in the cytoplasm (Fig. 1). Of the 57 melanoma tissues and 50 benign controls, LKB1 expression was demonstrated in 36 and 44 cases, respectively. In the 12 cases of LKB1-methylated melanoma, 6 cases demonstrated LKB1 expression. Notably, significant differences in LKB1 expression were observed between benign lesions with melanomas, and between LKB1 methylated melanomas and unmethylated melanoma ( $\mathrm{P}<0.05$, Kruskal-Wallis test; Fig. 2A and $\mathrm{B}$, respectively). In the melanoma cases, LKB1 protein expression determined by IHC was correlated with the status of LKB1 promoter methylation ( $r=0.473 ; \mathrm{P}<0.01$; data not shown).

Associations between LKB1 promoter methylation and clinicopathological factors. Patient information (age, sex, and location of melanoma) and clinicopathological parameters are detailed in Table II. Statistical analysis revealed that LKB1 promoter methylation was closely associated with tumor Breslow's thickness $(\mathrm{P}=0.021)$, the presence of ulceration $(\mathrm{P}=0.002)$ and AJCC stage $(\mathrm{P}=0.006)$. In the present study, no significant correlations between LKB1 promoter methylation and tumor location, age, sex or tumor subtype were observed (Table II). 
Table II. Association between LKB1 promoter methylation and different clinicopathological parameters in 57 melanoma cases.

\begin{tabular}{|c|c|c|c|c|c|}
\hline \multirow[b]{2}{*}{ Parameter } & \multirow[b]{2}{*}{$\mathrm{N}$} & \multicolumn{2}{|c|}{ LKB1 } & \multirow[b]{2}{*}{$\chi^{2}$} & \multirow[b]{2}{*}{ P-value } \\
\hline & & Methylated & Unmethylated & & \\
\hline Age at diagnosis & & & & 1.201 & 0.273 \\
\hline$\leq 60$ & 30 & 8 & 22 & & \\
\hline$>60$ & 27 & 4 & 23 & & \\
\hline Sex & & & & 0.030 & 0.863 \\
\hline Male & 32 & 7 & 25 & & \\
\hline Female & 25 & 5 & 20 & & \\
\hline Location & & & & 0.005 & 0.945 \\
\hline Sun-protected ${ }^{\mathrm{a}}$ & 28 & 6 & 22 & & \\
\hline Sun-exposed ${ }^{\mathrm{b}}$ & 29 & 6 & 23 & & \\
\hline Breslow's thickness, mm & & & & 5.291 & 0.021 \\
\hline$\leq 2$ & 31 & 3 & 28 & & \\
\hline$>2$ & 26 & 9 & 17 & & \\
\hline Ulceration & & & & 9.619 & 0.002 \\
\hline Absent & 32 & 2 & 30 & & \\
\hline Present & 25 & 10 & 15 & & \\
\hline Tumor subtype & & & & 1.679 & 0.642 \\
\hline ALM & 15 & 2 & 13 & & \\
\hline SSM & 12 & 2 & 10 & & \\
\hline LMM & 14 & 3 & 11 & & \\
\hline Mucosal & 16 & 5 & 11 & & \\
\hline AJCC stage & & & & 8.661 & 0.006 \\
\hline $\mathrm{I} / \mathrm{II}$ & 39 & 4 & 35 & & \\
\hline III/IV & 18 & 8 & 10 & & \\
\hline
\end{tabular}

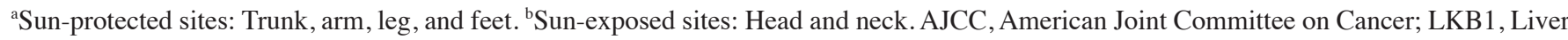
kinase B1; ALM, acral lentiginous melanoma; SSM, superficial spreading melanoma; LMM, lentigo maligna melanoma.

Table III. Multivariate Cox regression analysis of 5-year overall survival in melanoma patients with LKB1 promoter methylation.

\begin{tabular}{lccccc}
\hline Variable & $\mathrm{B}^{\mathrm{a}}$ & Standard error & P-value & Hazard ratio & 95\% confidence interval \\
\hline Age & 0.097 & 0.441 & 0.826 & 1.102 & $0.464-2.615$ \\
Sex & 0.495 & 0.383 & 0.196 & 1.641 & $0.774-3.478$ \\
Tumor location & -0.326 & 0.399 & 0.415 & 0.722 & $0.330-1.580$ \\
Breslow's thickness & -0.466 & 0.501 & 0.352 & 0.627 & $0.235-1.674$ \\
Ulceration & 1.02 & 0.481 & 0.034 & 2.773 & $1.081-7.111$ \\
Tumor subtype & -0.065 & 0.196 & 0.74 & 0.937 & $0.639-1.375$ \\
AJCC stage & -0.718 & 0.571 & 0.209 & 0.488 & $0.159-1.494$ \\
Methylation & 1.305 & 0.606 & 0.031 & 3.688 & $1.124-12.100$ \\
\hline
\end{tabular}

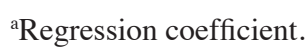

Prognostic analysis of melanoma patients with LKB1 promoter methylation. To investigate the prognostic significance of LKB1 promoter methylation in melanoma, Kaplan-Meier survival analysis and log-rank tests were used to assess the association between LKB1 promoter methylation and information on follow-up of patients. It was identified that patients with LKB1 methylation exhibited a significantly shorter overall 5-year OS duration compared with those with unmethylated LKB1 ( $\mathrm{P}<0.05$; Fig. 3). Concurrently, methylation and presence of ulceration were all associated with prognosis of CMM ( $\mathrm{P}<0.05$; Table III). Age, sex, location, Breslow's thickness and tumor subtype were not associated with prognosis of 

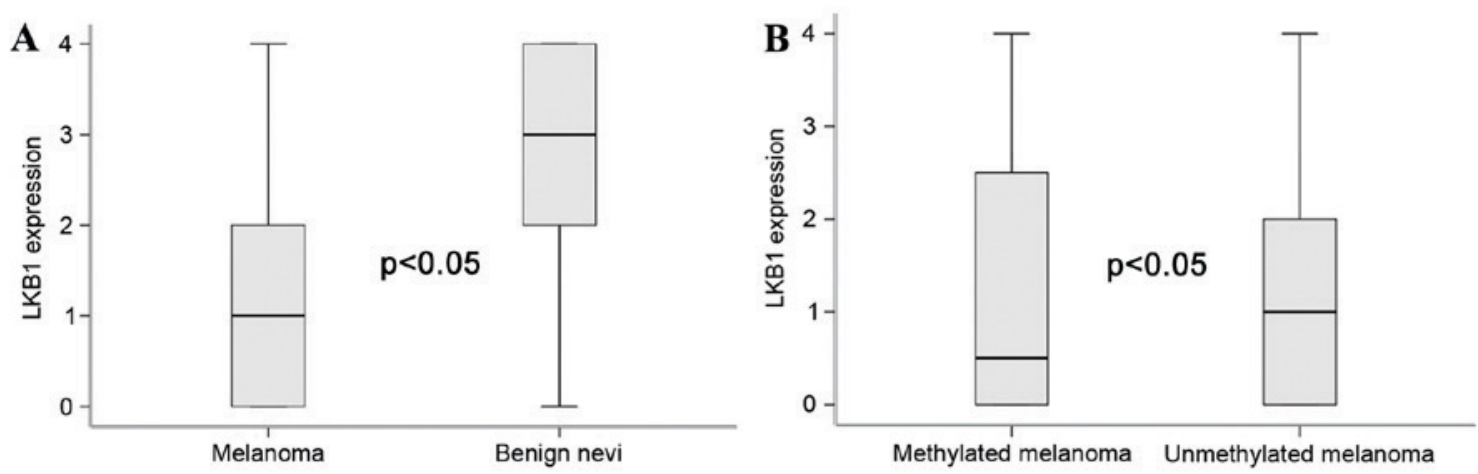

Figure 2. (A) Differences in LKB1 mRNA expression between melanoma and benign lesions. (B) Differences in LKB1 expression between LKB1 methylated melanoma and unmethylated melanoma ( $\mathrm{n}=57$; $\mathrm{P}<0.05$; Kruskal-Wallis test). LKB1, liver kinase B1.

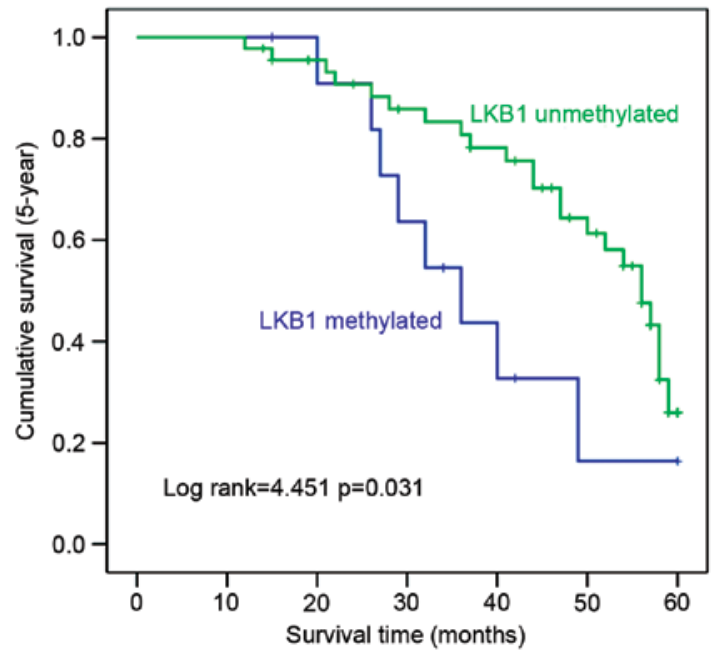

Figure 3. Kaplan-Meier survival curve of 5-year overall survival based on LKB1 promoter methylation status. LKB1, liver kinase B1.

CMM (P>0.05; Table III). Further multivariate Cox regression analysis indicated that LKB1 promoter methylation was an independent prognostic predictor of overall 5-year survival in melanoma patients (Table III).

\section{Discussion}

LKB1, also known as serine/threonine kinase, was identified as a crucial tumor suppressor gene in numerous types of tumors and cancer cells (4-5). Through activation of multiple signaling pathways, LKB1 functions to regulate cellular growth, energy metabolism and polarity in a number of tissues $(24,25)$. A previous study demonstrated that the loss of LKB1 serves an important role in tumor initiation and progression (26). At present, the loss of LKB1 in different human tumors is involved in a combination of three molecular mechanisms, including mutation, loss of heterozygosity $(\mathrm{LOH})$ and epigenetic modification (8). Although LKB1 gene mutation was hypothesized as the primary mechanism of LKBI inactivation in Peutz-Jeghers syndrome (PJS) or PJS-associated tumors, results from Avizienyte et al (27) suggested that mutational inactivation of LKB1 is a rare event in the majority of sporadic tumor types. As the primary epigenetic modification in humans, promoter methylation has been demonstrated in number of tumor and cell types. Trojan et al (28) confirmed that the 5'-CpG islands of the LKB1 promoter region were methylated when analyzing a large series of paraffin embedded colorectal cancer specimens. Esteller et al (29) also demonstrated LKB1 promoter methylation in primary colorectal cancer specimens and suggested that LKB1 promoter methylation may mediate the inactivation of LKB1.

To investigate the status of LKB1 promoter methylation in human cutaneous melanoma, the present study first examined LKB1 promoter methylation in melanoma tissues and benign skin lesion tissues by MSP. Of the 57 cases, 12 cases of melanoma tissues exhibited LKB1 promoter methylation, and the frequency of LKB1 promoter methylation in melanoma tissues was significantly increased compared with benign skin lesion tissues. The association between LKB1 promoter methylation and different clinicopathological parameters was investigated. It was demonstrated that LKB1 methylation status was significantly associated with Breslow's thickness $(\mathrm{P}<0.05)$, the presence of ulceration $(\mathrm{P}<0.01)$ and AJCC stage $(\mathrm{P}<0.01)$. The prognosis survival difference between the patients with LKB1 promoter methylation and the patients without methylation was also analyzed. The average duration of 5-year OS in patients with LKB1 methylation was significantly shorter compared with patients without LKB1 methylation. In addition, it was identified that LKB1 expression was markedly reduced in cutaneous melanoma tissues compared with benign skin lesions. In melanoma tissues, downregulated LKB1 expression was associated with its methylation status.

In conclusion, the results of the present study suggest that LKB1 gene promoter methylation in cutaneous melanoma was associated with clinicopathological parameters, and LKB1 may be an independent prognosis predicator. It was also demonstrated that downregulated LKB1 expression may be associated with LKB1 methylation. However, there are several limitations with the present study, including the use of a small sample size and a retrospective study design. The data need to be extensively evaluated in larger sample sizes and in multi-centric studies. The role of LKB1 mutations and $\mathrm{LOH}$ in cutaneous melanoma requires confirmation, and it is possible that LKB1 loss is caused by a combination of factors. 


\section{References}

1. Cockerell CJ: The pathology of melanoma. Dermatol Clin 30: 445-468, 2012.

2. Wisco OJ and Sober AJ: Prognostic factors for melanoma. Dermatol Clin 30: 469-485, 2012.

3. Tejera-Vaquerizo A, Solís-García E, Ríos-Martín JJ and Moreno-Ramírez D: Primary cutaneous melanoma: Prognostic factors not included in the classification of the american joint committee on cancer. Actas Dermosifiliogr 102: 255-263, 2011 (In Spanish).

4. Katajisto P, Vallenius T, Vaahtomeri K, Ekman N, Udd L, Tiainen M and Mäkelä TP: The LKB1 tumor suppressor kinase in human disease. Biochim Biophys Acta 1775: 63-75, 2007.

5. Hemminki A, Markie D, Tomlinson I, Avizienyte E, Roth S, Loukola A, Bignell G, Warren W, Aminoff M, Höglund P, et al: A serine/threonine kinase gene defective in Peutz-Jeghers syndrome. Nature 391: 184-187, 1998.

6. Li J, Liu J, Li P, Mao X, Li W, Yang J and Liu P: Loss of LKB1 disrupts breast epithelial cell polarity and promotes breast cancer metastasis and invasion. J Exp Clin Cancer Res 33: 70, 2014.

7. Zheng B, Jeong JH, Asara JM, Yuan YY, Granter SR, Chin L and Cantley LC: Oncogenic B-RAF negatively regulates the tumor suppressor LKB1 to promote melanoma cell proliferation. Mol Cell 33: 237-247, 2009 .

8. Gan RY and Li HB: Recent progress on liver kinase B1 (LKB1): Expression, regulation, downstream signaling and cancer suppressive function. Int J Mol Sci 15: 16698-16718, 2014.

9. Vaahtomeri K and Mäkelä TP: Molecular mechanisms of tumor suppression by LKB1. FEBS Lett 585: 944-951, 2011.

10. Partanen JI, Tervonen TA, Myllynen M, Lind E, Imai M, Katajisto P, Dijkgraaf GJ, Kovanen PE, Mäkelä TP, Werb Z and Klefström J: Tumor suppressor function of Liver kinase B1 (Lkb1) is linked to regulation of epithelial integrity. Proc Natl Acad Sci USA 109: E388-E397, 2012.

11. Zhou W, Zhang J and Marcus AI: LKB1 tumor suppressor: Therapeutic opportunities knock when LKB1 is inactivated. Genes Dis 1: 64-74, 2014

12. de Wilde RF, Ottenhof NA, Jansen M, Morsink FH, de Leng WW, Offerhaus GJ and Brosens LA: Analysis of LKB1 mutations and other molecular alterations in pancreatic acinar cell carcinoma. Mod Pathol 24: 1229-1236, 2012.

13. Lee SM, Choi JE, Na YK, Lee EJ, Lee WK, Choi YY, Yoon GS, Jeon HS, Kim DS and Park JY: Genetic and epigenetic alterations of the LKB1 gene and their associations with mutations in TP53 and EGFR pathway genes in Korean non-small cell lung cancers. Lung Cancer 81: 194-199, 2013.

14. Guldberg P, thor Straten P, Ahrenkiel V, Seremet T, Kirkin AF and Zeuthen J: Somatic mutation of the Peutz-Jeghers syndrome gene, LKB1/STK11, in malignant melanoma. Oncogene 18 : 1777-1780, 1999.

15. de Abreu FB, Gallagher TL, Liu EZ and Tsongalis GJ: Determining methylation status of methylguanine DNA methyl transferase (MGMT) from formalin-fixed, paraffin embedded tumor tissue. MethodsX 1: 42-48, 2014.
16. Ekizoglu S, Dalay N, Karaman E, Akdeniz D, Ozaydin A and Buyru N: LKB1 downregulation may be independent of promoter methylation or FOXO3 expression in head and neck cancer. Transl Res 162: 122-129, 2013.

17. He TY, Tsai LH, Huang CC, Chou MC and Lee H: LKB1 loss at transcriptional level promotes tumor malignancy and poor patient outcomes in colorectal cancer. Ann Surg Oncol 21 (Suppl 4): S703-S710, 2014.

18. Boland GM and Gershenwald JE: Principles of melanoma staging. Cancer Treat Res 167: 131-148, 2016.

19. Retsas S, Henry K, Mohammed MQ and MacRae K: Prognostic factors of cutaneous melanoma and a new staging system proposed by the American Joint Committee on Cancer (AJCC): Validation in a cohort of 1284 patients. Eur J Cancer 38: 511-516, 2002.

20. Moreno-Ramírez D, Ojeda-Vila T, Ríos-Martín JJ,Nieto-García A and Ferrándiz L: Association between tumor size and Breslow's thickness in malignant melanoma: A cross-sectional, multicenter study. Melanoma Res 25: 450-452, 2015.

21. Gown AM, Goldstein LC, Barry TS, Kussick SJ, Kandalaft PL, Kim PM and Tse CC: High concordance between immunohistochemistry and fluorescence in situ hybridization testing for HER2 status in breast cancer requires a normalized IHC scoring system. Mod Pathol 21: 1271-1277, 2008.

22. Kokkat TJ, Patel MS, McGarvey D, LiVolsi VA and Baloch ZW: Archived formalin-fixed paraffin-embedded (FFPE) blocks: A valuable underexploited resource for extraction of DNA, RNA, and protein. Biopreserv Biobank 11: 101-106, 2013.

23. Livak KJ and Schmittgen TD: Analysis of relative gene expression data using real-time quantitative PCR and the 2(-Delta Delta C(T)) Method. Methods 25: 402-408, 2001.

24. Chan KT, Asokan SB, King SJ, Bo T, Dubose ES, Liu W, Berginski ME, Simon JM, Davis IJ, Gomez SM, et al: LKB1 loss in melanoma disrupts directional migration toward extracellular matrix cues. J Cell Biol 207: 299-315, 2014.

25. Shin C and Tallon B: Assessment of tumor mitotic rate in primary cutaneous malignant melanomas $1 \mathrm{~mm}$ or less in thickness. J Am Acad Dermatol 72: 405-409, 2015.

26. Liu W, Monahan KB, Pfefferle AD, Shimamura T, Sorrentino J, Chan KT, Roadcap DW, Ollila DW, Thomas NE, Castrillon DH, et al: LKB1/STK11 Inactivation leads to expansion of a prometastatic tumor subpopulation in melanoma. Cancer Cell 21: 751-764, 2012.

27. Avizienyte E, Loukola A, Roth S, Hemminki A, Tarkkanen M, Salovaara R, Arola J, Bützow R, Husgafvel-Pursiainen K, Kokkola A, et al: LKB1 somatic mutations in sporadic tumors. Am J Pathol 154: 677-681, 1999.

28. Trojan J, Brieger A, Raedle J, Esteller M and Zeuzem S: 5'-CpG island methylation of the LKB1/STK11 promoter and allelic loss at chromosome $19 \mathrm{p} 13.3$ in sporadic colorectal cancer. Gut 47: 272-276, 2000.

29. Esteller M, Avizienyte E, Corn PG, Lothe RA, Baylin SB, Aaltonen LA and Herman JG: Epigenetic inactivation of LKB1 in primary tumors associated with the Peutz-Jeghers syndrome. Oncogene 19: 164-168, 2000 